\title{
COMPARATIVE PARAMETRIC STUDY OF LINEAR AND NONLINEAR BEHAVIOR OF MULTISTORY STRUCTURES
}

\author{
Mrunmayi Gursale ${ }^{1}$, P. S. Patil ${ }^{2}$ \\ ${ }^{I}$ Student M. Tech Structure, Rajarambapu Institute of Technology, Islampur \\ ${ }^{2}$ Professor Civil Engineering Dept, Rajarambapu Institute of Technology, Islampur
}

\begin{abstract}
The earthquake analysis of multistorey structure is done by linear and nonlinear methods. Response spectrum method of analysis is linear dynamic analysis. For nonlinear dynamic analysis time history method is used. In this paper, response spectrum method is used for linear analysis. For nonlinear analysis, time history method is used. For time history method, time history function of Bhuj earthquake is used. Both the analyses are done using ETABs software. To study seismic behaviour, base shear, time period, storey displacement parameters are studied
\end{abstract}

Keywords: linear analysis, nonlinear analysis, response spectrum method, time history method, ETABs.

\section{INTRODUCTION}

\subsection{General}

The earthquakes occurred in recent past have indicated that if structures are constructed without considering seismic forces, not properly designed, they will undergo huge destruction and also loss of life. So it is necessary to analyze structure for seismic responses. The analysis is performed on basis of external action, behaviour of structural material, type of structural model selected. Linear static analysis can be used for small structures. Linear dynamic analysis can be done by response spectrum method. In this method peak response of a structure is obtained directly from earthquake response.

Nonlinear method gives actual behaviour of structure. In nonlinear static analysis method, to study behaviour of structure, capacity curve is used. Capacity curve is relation between base shear and displacement of roof. It is also called Push over analysis. Nonlinear dynamic analysis is known as time history analysis. For this type of analysis, time history of a particular earthquake for a given structure is required. This method is used to determine seismic behaviour of structure under dynamic load.

The seismic responses and seismic performance of structure can be evaluated by dynamic response of equivalent single degree of freedom system obtained from multi degree of freedom system. The second method is obtaining responses by analysis of multi degree of freedom system.

\subsection{Linear Dynamic Analysis}

For linear dynamic analysis, response spectrum method is used. In this method, peak responses of a structure during the earthquake are obtained directly from earthquake responses. The response spectrum is an envelope of upper bound responses based on several different ground motions. The design spectrum given in IS 1893: 2002 Part 1 is used.
This spectrum is based on strong motion records of eight Indian earthquakes. In response spectrum method, maximum values of displacements and member forces are calculated for each mode, using design spectra. Response spectra help to calculate peak structural responses under linear range, which can be used to calculate lateral forces developed in structure due to earthquake. This helps for earthquake resistant design.

\subsection{Nonlinear Dynamic Analysis}

Nonlinear method is most accurate method for calculation of seismic responses of structure. For this type of analysis earthquake time history of representative earthquake is required. The base of the structure is subjected to actual ground motion which is representation of ground acceleration verses time. The ground acceleration is determined at small time steps to give ground motion record. In this dynamic analysis of structure is done at each increment of time. Three main parameters for time history are magnitude, distance and soil condition category. For time history analysis, recorded ground motion database from past natural events are used.

\section{MODELLING}

\subsection{General}

For parametric study of multistorey structure, ETABS software is used. Models for $\mathrm{G}+14, \mathrm{G}+17$, and $\mathrm{G}+20$ are generated. These models are analysed by response spectrum and time history method. ETABS is completely integrated software for analysis and design of reinforced structure. Creation of model, modification of model, analysis, all are accomplished using single interface. A single structural model can be used for wide variety of different type analysis and design. 


\subsection{Problem Statement}

Models for $\mathrm{G}+14, \mathrm{G}+17$ and $\mathrm{G}+20$ are taken for purpose of study. These models are analysed by both response spectrum and time history method. For time history analysis method, time history record of Bhuj earthquake is used. The typical floor height is taken as $3 \mathrm{~m}$.

\subsubsection{Dimensions of Proposed Model}

Table 2.1 Dimensions of Model

\begin{tabular}{|l|l|l|}
\hline $\begin{array}{l}\text { Sr. } \\
\text { No. }\end{array}$ & Property & Dimension \\
\hline 1 & Plan Dimension in X Direction & $16 \mathrm{~m}$ \\
\hline 2 & Plan Dimension in Y Direction & $12 \mathrm{~m}$ \\
\hline
\end{tabular}

\subsubsection{Loads Considered as per IS 1893 - 2002 (Part}

I)

1. Dead Load

2. Live Load $=2 \mathrm{kN} / \mathrm{m}^{2}$

3. Floor Finish $=1 \mathrm{kN} / \mathrm{m}^{2}$

4. Earthquake force in $\mathrm{X}$ direction

5. Earthquake force in $Y$ direction

6. Wind force in $\mathrm{X}$ direction

7. Wind force in $\mathrm{Y}$ direction

\subsubsection{Earthquake Parameters Based on Structure}

\section{Location IS 1893 - 2002 (Part I)}

Table 2.2 Earthquake Parameters Based on Structure Location IS 1893 - 2002 (Part I)

\begin{tabular}{|l|l|l|}
\hline Sr. No. & Parameters & Code Provisions \\
\hline 1 & Type of Structure & RCC \\
\hline 2 & Nature of Building & Residential \\
\hline 3 & Damping of Concrete & $5 \%$ \\
\hline
\end{tabular}

\subsection{Structural Modelling and Analysis}
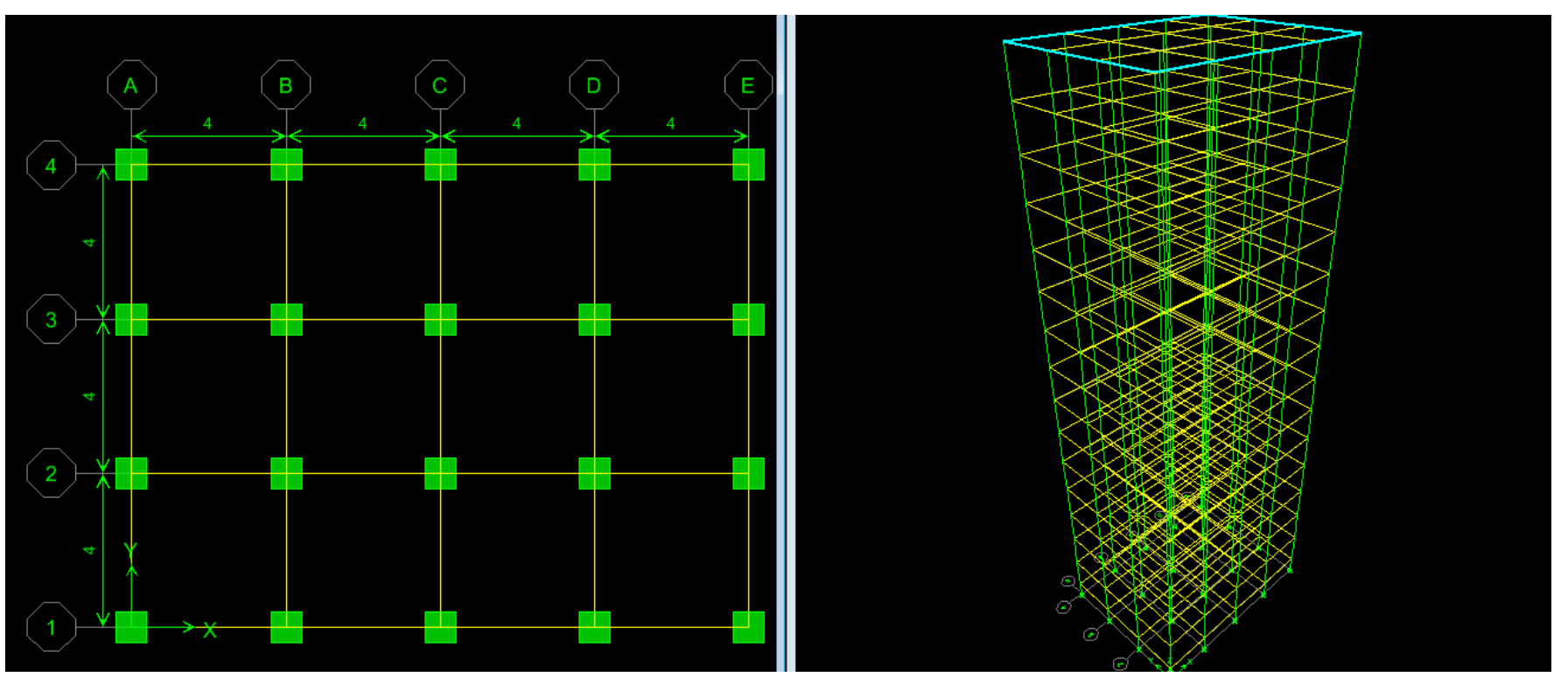

Fig.1 Model of G+ 14 Structures

Table 2.3 Sizes of Structural Elements

\begin{tabular}{|l|l|l|}
\hline Sr. No. & Structural Member & Size \\
\hline 1 & Columns & $800 \mathrm{mmX800mm}$ \\
\hline 2 & Beams & $350 \mathrm{mmX600mm}$ \\
\hline 3 & $\begin{array}{l}\text { Thickness of typical } \\
\text { slab }\end{array}$ & $125 \mathrm{~mm}$ \\
\hline 4 & Thickness of wall & $230 \mathrm{~mm}$ \\
\hline 5 & $\begin{array}{l}\text { Grade of Concrete and } \\
\text { Steel }\end{array}$ & M20 \& Fe415 \\
\hline
\end{tabular}

\subsubsection{Seismic and Wind Parameters}

Table 2.4 Seismic and Wind Parameters

\begin{tabular}{|l|l|l|l|l|}
\hline Sr. No. & Location & $\begin{array}{l}\text { Seismic } \\
\text { Zone }\end{array}$ & $\begin{array}{l}\text { Zone } \\
\text { Factor }(\mathrm{Z})\end{array}$ & $\begin{array}{l}\text { Wind } \\
\text { Speed m/s) }\end{array}$ \\
\hline 1 & Bhuj & $\mathrm{V}$ & 0.36 & 50 \\
\hline
\end{tabular}

\subsubsection{Sizes of Structural Members}



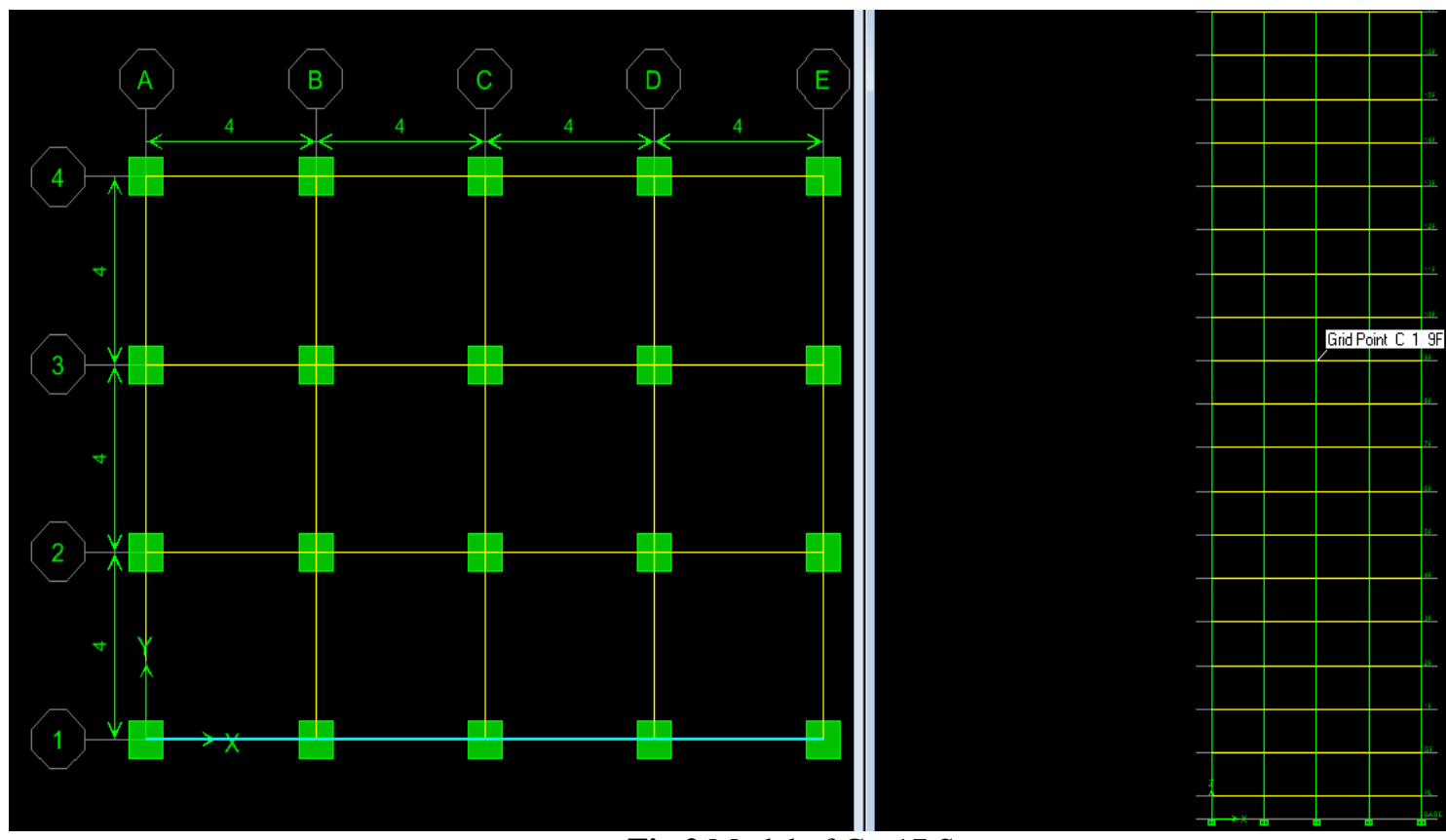

Fig.2 Model of G+ 17 Structures
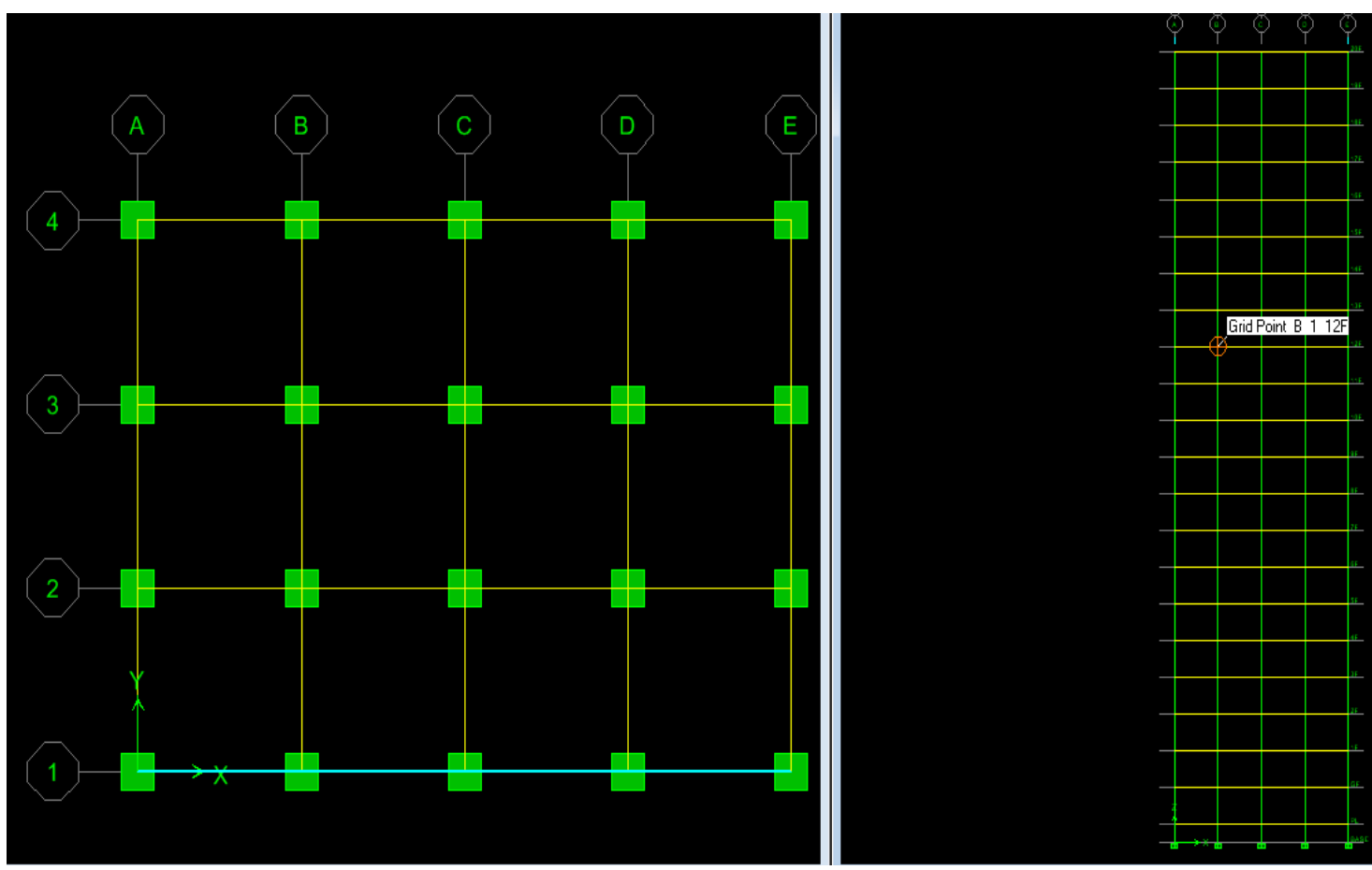

Fig.3 Model of G+ 20 Structures

\section{RESULTS AND DISCUSSION}

The results of analysis of multistorey structure by both linear and nonlinear methods are tabulated below.

\subsection{Linear Analysis (Response Spectrum Method)}

3.1.1 Following Table shows values of Storey Shear for G+14, G+17, G+20 Structures.

Table 3.1 Storey Shear of G+14, G+17, G+20 Structures

\begin{tabular}{|l|l|l|l|}
\hline Floor & $\begin{array}{l}\text { Storey Shear } \\
\text { G+20 Structure }\end{array}$ & $\begin{array}{l}\text { Storey Shear }(\mathrm{KN}) \text { for } \\
\text { G+17 Structure }\end{array}$ & $\begin{array}{l}\text { Storey Shear }(\mathrm{KN}) \text { for } \\
\text { G+14 Structure }\end{array}$ \\
\hline $20 \mathrm{~F}$ & 147.03 & - & - \\
\hline $19 \mathrm{~F}$ & 328.98 & - & - \\
\hline
\end{tabular}




\begin{tabular}{|l|l|l|l|}
\cline { 2 - 4 } $18 \mathrm{~F}$ & 487.04 & - & - \\
\hline $17 \mathrm{~F}$ & 619.06 & 156.11 & - \\
\hline $16 \mathrm{~F}$ & 726.99 & 346.99 & - \\
\hline $15 \mathrm{~F}$ & 815.36 & 510.5 & - \\
\hline $14 \mathrm{~F}$ & 888.73 & 646.49 & 169.47 \\
\hline $13 \mathrm{~F}$ & 950.35 & 759.71 & 373.84 \\
\hline $12 \mathrm{~F}$ & 1002.49 & 855.94 & 547.42 \\
\hline $11 \mathrm{~F}$ & 1047.79 & 939.07 & 693.89 \\
\hline $10 \mathrm{~F}$ & 1089.96 & 1011.47 & 820.38 \\
\hline $9 \mathrm{~F}$ & 1132.94 & 1076.11 & 931.51 \\
\hline $8 \mathrm{~F}$ & 1179.32 & 1137.1 & 1029.58 \\
\hline $7 \mathrm{~F}$ & 1229.63 & 1197.8 & 1117.81 \\
\hline $6 \mathrm{~F}$ & 1282.98 & 1259.2 & 1200.17 \\
\hline $5 \mathrm{~F}$ & 1338.2 & 1320.36 & 1278.26 \\
\hline $4 \mathrm{~F}$ & 1393.96 & 1380.04 & 1351.01 \\
\hline $3 \mathrm{~F}$ & 1447.68 & 1436.72 & 1416.94 \\
\hline $2 \mathrm{~F}$ & 1494.68 & 1486.89 & 1474.04 \\
\hline $1 \mathrm{~F}$ & 1529.04 & 1524.49 & 1517.31 \\
\hline GF & 1546.53 & 1544.08 & 1540.36 \\
\hline $\mathrm{PL}$ & 1548.08 & 1545.83 & 1542.45 \\
\hline
\end{tabular}

\subsubsection{Storey Displacement}

Table 3.4 Storey Displacement of $\mathrm{G}+14, \mathrm{G}+17, \mathrm{G}+20$ Structures

\begin{tabular}{|l|l|l|l|}
\hline Floor & $\begin{array}{l}\text { Storey Displacement }(\mathrm{m}) \\
\text { G+20 Structure }\end{array}$ & $\begin{array}{l}\text { Storey Displacement }(\mathrm{m}) \\
\text { G+17 Structure }\end{array}$ & $\begin{array}{l}\text { Storey Displacement }(\mathrm{m}) \\
\text { G+14 Structure }\end{array}$ \\
\hline $20 \mathrm{~F}$ & 0.0386 & - & - \\
\hline $19 \mathrm{~F}$ & 0.0378 & - & - \\
\hline $18 \mathrm{~F}$ & 0.0369 & - & - \\
\hline $17 \mathrm{~F}$ & 0.0359 & 0.0321 & - \\
\hline $16 \mathrm{~F}$ & 0.0346 & 0.0315 & - \\
\hline $15 \mathrm{~F}$ & 0.0333 & 0.0306 & - \\
\hline $14 \mathrm{~F}$ & 0.0318 & 0.0296 & 0.0259 \\
\hline $13 \mathrm{~F}$ & 0.0301 & 0.0284 & 0.0253 \\
\hline $12 \mathrm{~F}$ & 0.0284 & 0.027 & 0.0245 \\
\hline $11 \mathrm{~F}$ & 0.0266 & 0.0255 & 0.0234 \\
\hline $10 \mathrm{~F}$ & 0.0247 & 0.0238 & 0.0222 \\
\hline $9 \mathrm{~F}$ & 0.0227 & 0.022 & 0.0208 \\
\hline $8 \mathrm{~F}$ & 0.0206 & 0.0201 & 0.0191 \\
\hline $7 \mathrm{~F}$ & 0.0184 & 0.018 & 0.0174 \\
\hline $6 \mathrm{~F}$ & 0.0162 & 0.0159 & 0.0154 \\
\hline $5 \mathrm{~F}$ & 0.0139 & 0.0137 & 0.0133 \\
\hline $4 \mathrm{~F}$ & 0.0115 & 0.0114 & 0.0111 \\
\hline $3 \mathrm{~F}$ & 0.0091 & 0.009 & 0.0088 \\
\hline $2 \mathrm{~F}$ & 0.0066 & 0.0065 & 0.0064 \\
\hline $1 \mathrm{~F}$ & 0.0042 & 0.0041 & 0.0041 \\
\hline $\mathrm{GF}$ & 0.0019 & 0.0019 & 0.0019 \\
\hline
\end{tabular}

\subsubsection{Time Period}

Table 3.5 Time Period of $\mathrm{G}+14, \mathrm{G}+17, \mathrm{G}+20$ Structures

\begin{tabular}{|l|l|l|}
\hline Sr. No. & Model & Time Period (sec) \\
\hline 1. & G+14 & 1.22 \\
\hline 2. & G+17 & 1.5 \\
\hline 3. & G+20 & 1.81 \\
\hline
\end{tabular}




\subsection{Nonlinear Analysis (Time History Method)}

\subsubsection{Storey Shear}

Table 3.6 Storey Shear of G+14, G+17, G+20 Structures

\begin{tabular}{|l|l|l|l|}
\hline Floor & $\begin{array}{l}\text { Storey Shear }(\mathrm{KN}) \text { for } \\
\text { G+20 Structure }\end{array}$ & $\begin{array}{l}\text { Storey Shear } \\
\text { G+17 Structure }\end{array}$ & $\begin{array}{l}\text { Storey Shear (KN) for } \\
\text { G+14 Structure }\end{array}$ \\
\hline $20 \mathrm{~F}$ & 61.90 & - & - \\
\hline $19 \mathrm{~F}$ & 140.24 & - & - \\
\hline $18 \mathrm{~F}$ & 214.25 & - & - \\
\hline $17 \mathrm{~F}$ & 272.51 & 68.72 & - \\
\hline $16 \mathrm{~F}$ & 340.96 & 162.74 & - \\
\hline $15 \mathrm{~F}$ & 448.45 & 280.78 & - \\
\hline $14 \mathrm{~F}$ & 538.04 & 391.39 & 71.35 \\
\hline $13 \mathrm{~F}$ & 661.35 & 528.68 & 159.37 \\
\hline $12 \mathrm{~F}$ & 710.77 & 606.86 & 240.81 \\
\hline $11 \mathrm{~F}$ & 749.27 & 671.53 & 305.45 \\
\hline $10 \mathrm{~F}$ & 785.86 & 729.27 & 384.76 \\
\hline $9 \mathrm{~F}$ & 837.13 & 795.14 & 512.33 \\
\hline $8 \mathrm{~F}$ & 880.01 & 848.50 & 623.31 \\
\hline $7 \mathrm{~F}$ & 934.40 & 910.21 & 777.88 \\
\hline $6 \mathrm{~F}$ & 978.14 & 960.01 & 915.01 \\
\hline $5 \mathrm{~F}$ & 1067.75 & 1053.52 & 1019.92 \\
\hline $4 \mathrm{~F}$ & 1088.46 & 1086.87 & 1082.43 \\
\hline $3 \mathrm{~F}$ & 1093.24 & 1091.51 & 1084.50 \\
\hline $2 \mathrm{~F}$ & 1109.78 & 1105.69 & 1088.88 \\
\hline $1 \mathrm{~F}$ & 1116.84 & 1106.47 & 1101.26 \\
\hline $\mathrm{GF}$ & 1126.84 & 1120.97 & 1108.05 \\
\hline $\mathrm{PL}$ & 1132.09 & 1123.52 & 1111.28 \\
\hline
\end{tabular}

\subsubsection{Storey Displacement}

Table 3.7 Storey Displacement of $\mathrm{G}+14, \mathrm{G}+17, \mathrm{G}+20$ Structures

\begin{tabular}{|l|l|l|l|}
\hline Floor & $\begin{array}{l}\text { Storey Displacement }(\mathrm{m}) \\
\text { for G+20 Structure }\end{array}$ & $\begin{array}{l}\text { Storey Displacement } \\
\text { for G+17 Structure }\end{array}$ & $\begin{array}{l}\text { Storey Displacement (m) } \\
\text { for G+14 Structure }\end{array}$ \\
\hline $20 \mathrm{~F}$ & 0.0304 & - & - \\
\hline $19 \mathrm{~F}$ & 0.0295 & - & - \\
\hline $18 \mathrm{~F}$ & 0.0288 & - & - \\
\hline $17 \mathrm{~F}$ & 0.0280 & 0.0250 & - \\
\hline $16 \mathrm{~F}$ & 0.0264 & 0.0240 & - \\
\hline $15 \mathrm{~F}$ & 0.0253 & 0.0233 & - \\
\hline $14 \mathrm{~F}$ & 0.0241 & 0.0224 & 0.0196 \\
\hline $13 \mathrm{~F}$ & 0.0223 & 0.0210 & 0.0187 \\
\hline $12 \mathrm{~F}$ & 0.0202 & 0.0192 & 0.0174 \\
\hline $11 \mathrm{~F}$ & 0.0179 & 0.0172 & 0.0158 \\
\hline $10 \mathrm{~F}$ & 0.0155 & 0.0150 & 0.0140 \\
\hline $9 \mathrm{~F}$ & 0.0131 & 0.0127 & 0.0120 \\
\hline $8 \mathrm{~F}$ & 0.0108 & 0.0105 & 0.0100 \\
\hline $7 \mathrm{~F}$ & 0.0091 & 0.0089 & 0.0086 \\
\hline $6 \mathrm{~F}$ & 0.0079 & 0.0078 & 0.0075 \\
\hline $5 \mathrm{~F}$ & 0.0067 & 0.0066 & 0.0064 \\
\hline $4 \mathrm{~F}$ & 0.0055 & 0.0054 & 0.0053 \\
\hline $3 \mathrm{~F}$ & 0.0041 & 0.0040 & 0.0039 \\
\hline $2 \mathrm{~F}$ & 0.0029 & 0.0029 & 0.0028 \\
\hline $1 \mathrm{~F}$ & 0.0018 & 0.0018 & 0.0018 \\
\hline $\mathrm{GF}$ & 0.0008 & 0.0008 & 0.0008 \\
\hline
\end{tabular}


Following figures show the comparative storey shear graph by both response spectrum and time history method for G+ 14, G+17, $\mathrm{G}+20$ structures.

\section{Storey Shear in KN for G+14}

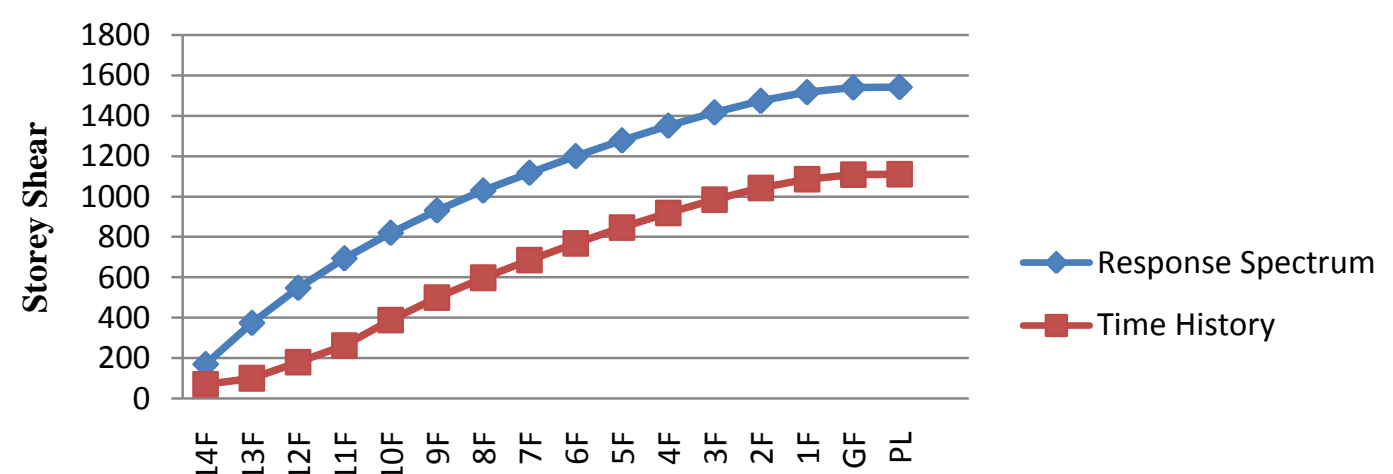

Floor

Fig. 4 Storey Shear in KN for G+14 Structure

\section{Storey Shear in KN for $\mathbf{G + 1 7}$}

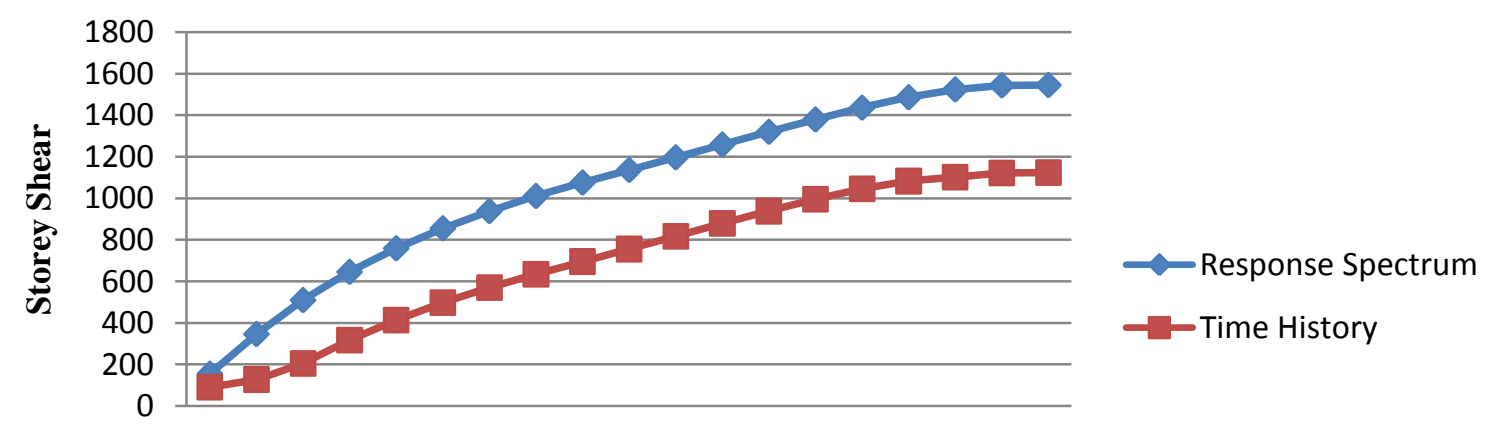

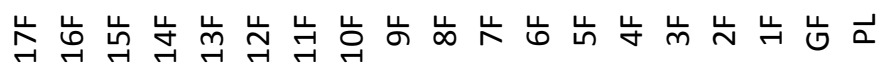

Floor

Fig. 5 Storey Shear in KN for G+17 Structure

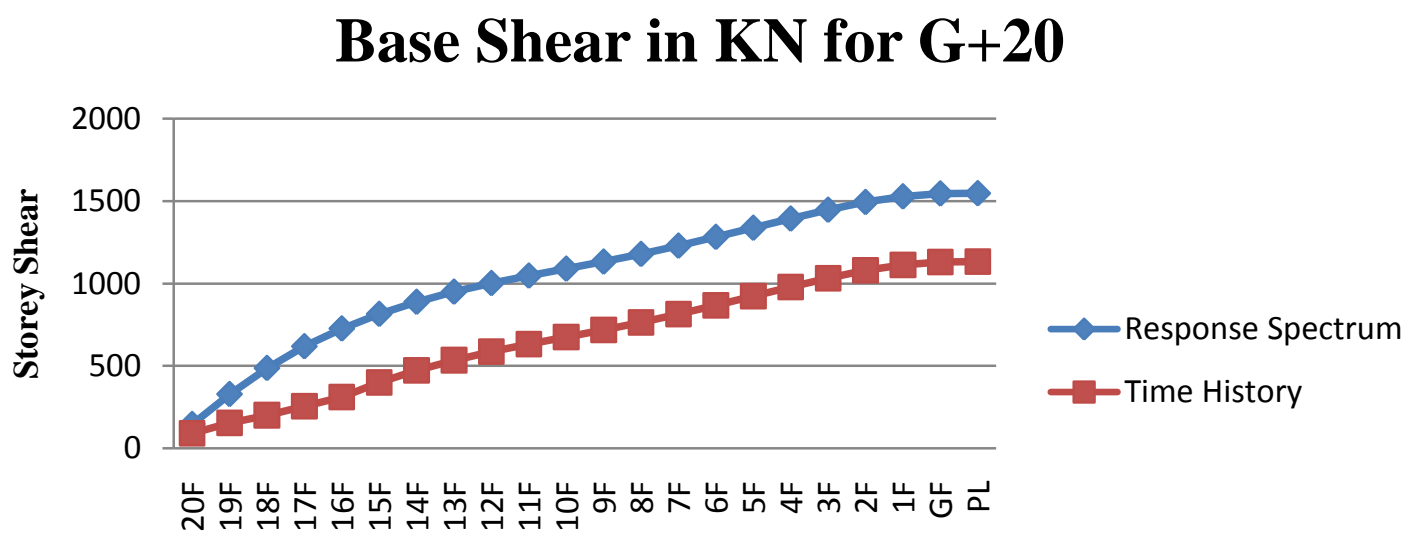

Floor

Fig. 6 Storey Shear in KN for G+20 Structure 
Following figures show the comparative storey shear graph by both response spectrum and time history method for G+ 14, G+17, $\mathrm{G}+20$ structures.

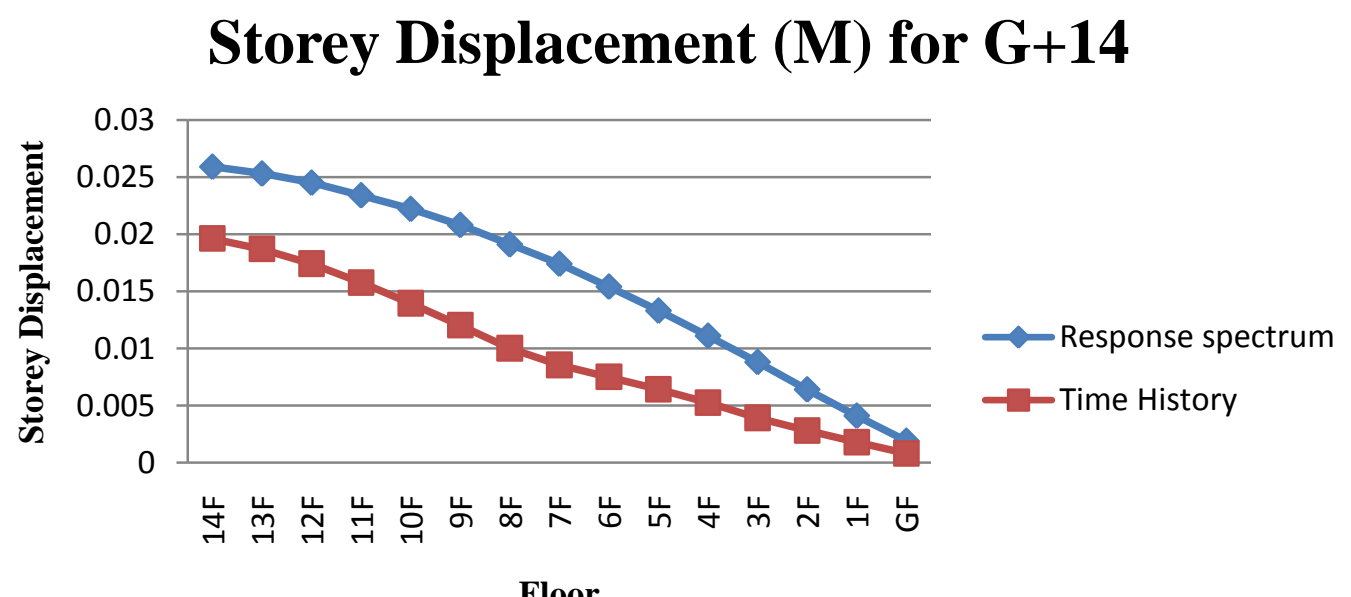

Fig. 7 Storey Displacement in $\mathrm{M}$ for $\mathrm{G}+14$ structure

\section{Storey Displacement (M) for $\mathbf{G + 1 7}$}

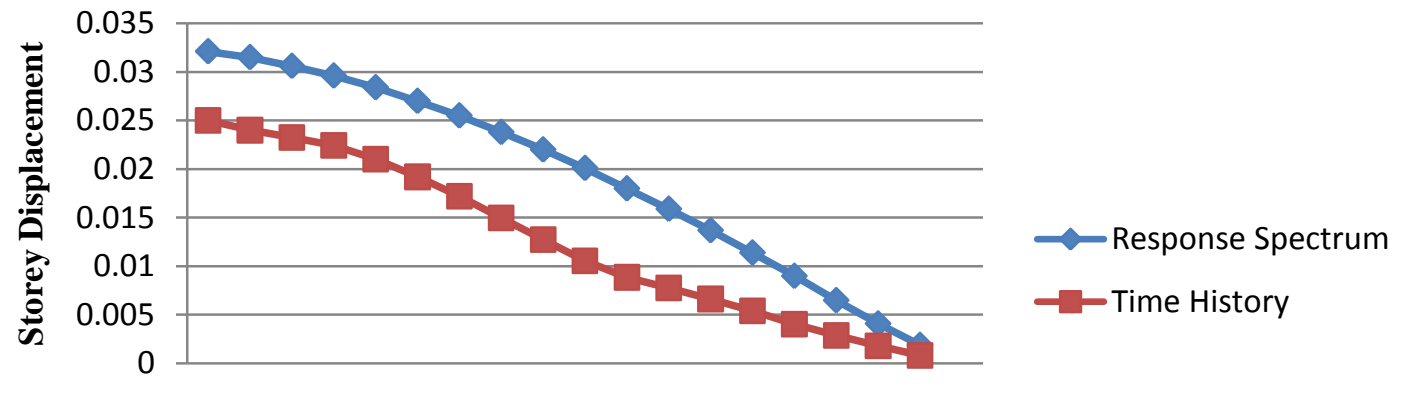

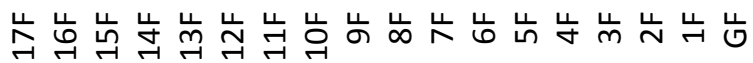

Floor

Fig. 8 Storey Displacement in M for G+17 Structure

Storey Displacement (M) for $\mathbf{G + 2 0}$

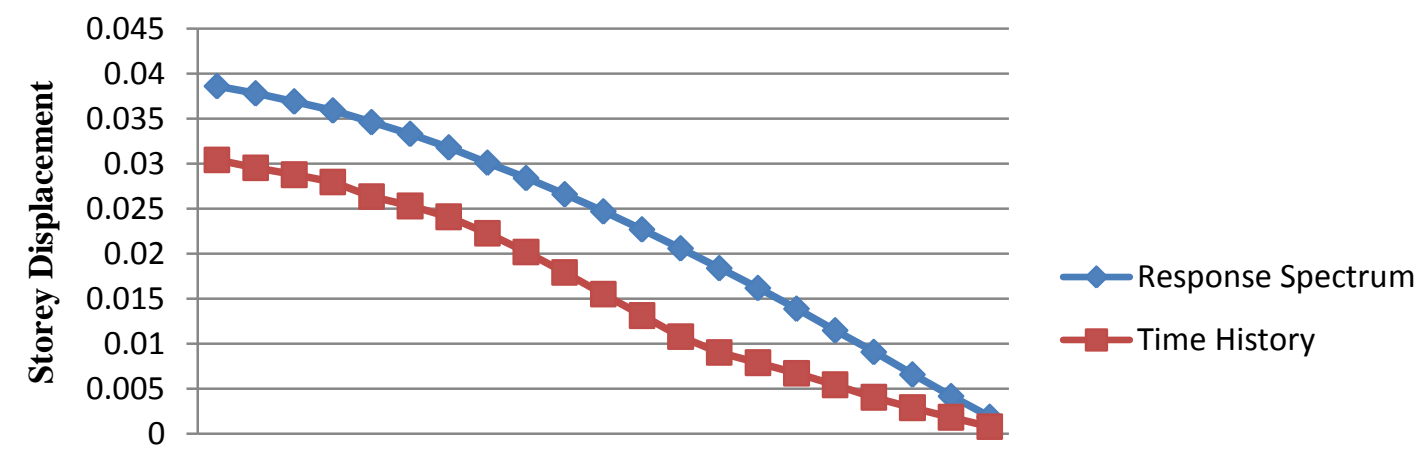

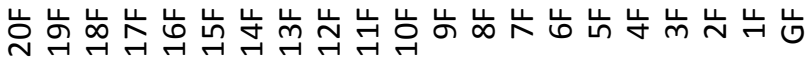

Floor

Fig. 9 Storey Displacement in M for G+20 Structure 


\section{CONCLUSION}

The study is based on linear and nonlinear analysis of multistorey structure for finding base shear, storey displacement and time period.

1. The storey shear results obtained for $\mathrm{G}+14, \mathrm{G}+17$, and $\mathrm{G}+$ 20 by response spectrum method are respectively $37 \%$, $30.63 \%$, and $34.24 \%$ more than those obtained by time history method.

2. The storey displacement results obtained for $\mathrm{G}+14, \mathrm{G}+17$, and $\mathrm{G}+20$ by response spectrum method are respectively $44.59 \%, 30.63 \%$, and $34.24 \%$ more than those obtained by time history method.

3. From results it is observed that time period of structure increases with increase in height.

4. As base shear depends on weight of structure, it is observed that it increases with increase in height both by response spectrum and time history method.

4. From results it is recommended that time history analysis should be performed as it predicts the structural response more accurately than response spectrum analysis.

\section{REFERENCES}

[1] Colin M. Morison, "Dynamic Response of Walls and Slabs by Single Degree of Freedom Analysis - A Critical Review and Revision", International Journal of Impact Engineering, Elsevier, 2005.

[2] Dang - Guen Lee, Won - Ho Choi, "Evaluation of Seismic performance of multistory building structures based on the equivalent responses" Engineering Structures, Elsevier, 28, 2006.

[3] Paolo Foraboschi, Alessia Vanin, "Nonlinear Static Analysis of Masonry Buildings Based on a Strut and Tie Method", Soil Dynamics and Earthquake Engineering 55, Elsevier, October 2013

[4] Raja Rizwan Hussain, Mohammed Jameel, "Nonlinear time domain analysis of base isolated multistory building under site specific bi - directional seismic loading" Automation in Construction, Elsevier, 22, December 2011.

[5] Reza Sehhati, Adrian Rodriguez, "Effects of near fault ground motions and equivalent pulses on multistory structure" Engineering structures, Elsevier, 33, January 2011.

[6] S. M. Wilkinson and R. A. Hiley, "A nonlinear time history model for the seismic analysis of high rise framed structures" Computers and Structures, Elsevier, 84, 2006.

[7] Victor I. Fernandez - Davila, Ernesto F. Cruz, "Parametric Study of nonlinear seismic response of three dimensional building models" Engineering Structures, Elsevier, 28, 2006. 